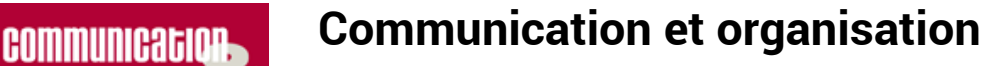

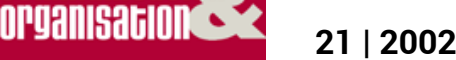

Espace et communication dans les organisations

\title{
Les bureaux ne sont pas seulement des espaces de travail...
}

Anne Monjaret

\section{OpenEdition}

1 Journals

Édition électronique

URL : http://journals.openedition.org/communicationorganisation/2645

DOI : 10.4000/communicationorganisation.2645

ISSN : 1775-3546

Éditeur

Presses universitaires de Bordeaux

Édition imprimée

Date de publication : 1 mai 2002

ISSN : 1168-5549

Référence électronique

Anne Monjaret, « Les bureaux ne sont pas seulement des espaces de travail... », Communication et organisation [En ligne], 21 | 2002, mis en ligne le 27 mars 2012, consulté le 10 décembre 2020. URL http://journals.openedition.org/communicationorganisation/2645; DOI : https://doi.org/10.4000/ communicationorganisation. 2645

Ce document a été généré automatiquement le 10 décembre 2020.

(c) Presses universitaires de Bordeaux 


\title{
Les bureaux ne sont pas seulement des espaces de travail...
}

\author{
Anne Monjaret
}

1 Comme nous l'écrivions déjà en 1996 dans un article consacré aux différents modes d'appropriation dans les bureaux, les architectes, les aménageurs, les ergonomes, les designers, les producteurs de mobilier ont examiné depuis longtemps les espaces de travail et leur physionomie. À l'occasion de programmations d'immeubles, ces acteurs se trouvent réunis. Les responsables de direction qui seront en poste dans les futurs locaux sont associés aux prérogatives. Les sociologues, eux, peuvent être sollicités lors des phases d'aménagement.

2 Les mouvements de l'immobilier, l'architecture externe et interne des locaux, le cadre de travail (ses formes, ses couleurs et ses matériaux), le mobilier et ses incidences sur le bien-être des employés sont autant de thèmes abordés par ces spécialistes. Si, en France, les sciences sociales s'y sont penchées, on doit cet intérêt aux psychosociologues (Fischer, 1989) et aux sociologues de l'organisation qui ont cherché à cerner les effets de cet environnement sur le travail. Les ethnologues habitués à l'étude des ateliers découvrent depuis peu le secteur tertiaire, parmi lesquels Moulinié (1993), Guigo (1994). Leurs approches restent encore trop isolées. Il s'agit donc d'entreprendre une anthropologie des « organisations modernes » (entreprise, administration, institution de service) pour rendre compte de leurs cultures matérielles et symboliques.

3 Les établissements professionnels doivent se lire dans la multiplicité de leurs temps, ordinaires et extra-ordinaires, et de leurs espaces, de travail (les bureaux, les salles de réunion), de passage et de circulation (le hall d'entrée, les couloirs), de restauration et de détente (restaurant d'entreprise, cafétérias). Nous avons choisi ici de présenter l'un de ces derniers : le bureau. On peut se demander en quoi il est pertinent de s'intéresser à un tel objet d'étude. Sans être exhaustif, deux raisons peuvent être avancées pour expliciter ce choix : la première est que les travaux ethnologiques en font aujourd'hui l'impasse, la seconde est que ce lieu incarne à la fois un passé administratif à l'image poussiéreuse et, paradoxalement, un présent et un futur tournés vers le progrès, une communication rapide possible grâce aux nouvelles technologies (fax, informatique-internet, téléphone 
portable) qui changent les données du travail et les rapports entre les sphères professionnelles et privées, on va vers une toujours plus grande imbrication ${ }^{1}$. En outre, à un moment où les questions de société se polarisent autour des thèmes de la mobilité, du télétravail ou de la R.T.T., son étude contribue, en contrepoint, à évaluer l'inscription territoriale des salariés pleinement ou partiellement sédentaires, au travail, en utilisant les objets qui les entourent comme indicateur d'un potentiel ancrage.

4 Partir de l'analyse environnementale des employés, de leur unité écologique, c'est comprendre comment ces derniers évoluent au quotidien dans leur univers professionnel, c'est aussi considérer que l'espace au travail est non seulement un espace de travail mais plus largement aussi un lieu de vie. En effet, nous concevons les bureaux comme des espaces occupés, habités ${ }^{2}$, vécus par les salariés, ce qui sous-entend qu'ils ne s'y exercent pas uniquement des activités professionnelles (Nippert-Eng, 1996).

5 Cet article ${ }^{3}$ examine, dans une perspective historique et sociologique ${ }^{4}$, comment les employés occupent leur bureau, comment ils fabriquent un territoire plus ou moins protégé, plus ou moins partagé. Les objets et les usages (légaux, tolérés ou interdits) quotidiens sont, pour nous, les indices de formalisation de cette occupation et d'élaboration d'un bien-être. À travers les marquages institutionnels et individuels ainsi que les modes d'appropriation et leur reproduction normative, nous chercherons à saisir l'organisation sociale des établissements professionnels. On peut se demander en quoi ces pratiques sont favorables à la communication entre salariés, participent au développement du lien social sur les lieux de travail, et par là à la construction des identités professionnelles et sociales. De même, en quoi les mises en scène types dans ces locaux peuvent-elles sous-entendre l'existence d'une culture des bureaux? Telles sont les questions auxquelles nous tenterons de répondre.

\section{Les bureaux : de l'habitation à l'immeuble spécialisé}

6 Les bureaux n'ont pas toujours été installés dans des immeubles spécialisés. C'est au XVIII e siècle, comme le décrit Natacha Coquery (1997), que s'amorce la conversion des hôtels particuliers aristocratiques qui servaient à la fois de logement et de lieu de travail, en bureaux aux aménagements normalisés et rationalisés dont l'uniformité devait exprimer l'égalité. On assiste ainsi au passage de la propriété privée à la propriété publique. Vient ensuite la construction de bâtiments administratifs, à l'architecture monumentale, symbole du pouvoir tout puissant de l'État.

7 Le XIX ${ }^{e}$ siècle signe l'amplification de ce phénomène bureaucratique mais les lieux de travail plus communs, plus diversifiés aussi, restent installés dans des bâtiments à double fonction : "Au début du siècle, un même immeuble offre à la fois des habitations et des lieux de travail dans une véritable continuité urbaine, les bureaux se situent de préférence au rez-dechaussée et a l'entresol (...) Les besoins en bureaux restent modestes » (Pélegrin-Genel, 1995 : 26). En dehors des bâtiments administratifs d'État, il faut attendre le Second Empire pour voir se développer la demande en espace de travail, afférente entre autres à l'expansion du système bancaire. Un quartier d'affaires émerge entre l'opéra et la Bourse.

$8 \mathrm{Au} \mathrm{XX}^{\mathrm{e}}$ siècle, les immeubles de bureaux se font toujours plus nombreux suivant l'essor du secteur tertiaire qui connaît une forte expansion dans les années 1960. Les années 1970 sont marquées par la modernisation de ce secteur dont les activités se diversifient. L'émergence de l'informatisation et de la télécommunication conduit à l'édification 
d'immeubles spécialisés et encourage les sièges sociaux à s'installer hors des sites de production. Ces exigences technologiques dictent les configurations spatiales. De nouvelles conceptions managériales se mettent en place avec l'aménagement des bureaux paysages (open space) qui rappellent étrangement les salles de pool de dactylos du début $\mathrm{du} \mathrm{XX}^{\mathrm{e}}$ siècle.

De nouveaux quartiers d'affaire surgissent comme ceux de La Défense et de Cergy Pontoise. L'augmentation des loyers parisiens et de la taxe professionnelle a incité certaines entreprises à sortir de Paris. Le parc immobilier s'étend jusqu'à la saturation observée ces dernières années. L'externalisation des activités de service autant que l'usage d'instruments de travail tels le téléphone et l'ordinateur portables qui ont contribué à la mobilité des salariés (Lautier, 2000), participent à une diminution des besoins . Durant les années 1990, les bureaux désertés sont réhabilités en appartement. Les boutiques de quartier, anciens commerces, sont métamorphosées en bureau.

10 Au cours des siècles, les bâtiments de bureaux se sont donc modifiés, et avec eux, leur morphologie spatiale interne (clos, paysage, caisson). Toutes ces transformations impliquent de nouvelles conditions de travail et permettent de révéler les changements de politique de gestion dans les administrations et les entreprises.

\section{Poser le décor : à l'origine des bureaux}

11 Le bureau n'a pas toujours été le lieu de travail que l'on connaît aujourd'hui. «Le mot "bureau " vient certainement de "bure", grosse toile de laine qui. placée sur les tables à écrire, permettait par son épaisseur et sa matière d'isoler le parchemin sur lequel on écrivait, évitant ainsi de le détériorer » (Rheims, 1990 : 1108). Ainsi, " par métonymies successives, on est passé dudit tapis de table à la table à écrire elle-même, puis de ladite table à la pièce dans laquelle elle était installée, puis à l'ensemble des meubles constituant cette pièce, et enfin aux activités qui s'y exercent, aux pouvoirs qui s'y rattachent, voire même aux services qui s'y rendent » (Perec, 1989 : 89).

On doit la physionomie moderne du bureau au développement de l'administration. Cette pièce se définit par sa culture matérielle que l'on pourrait qualifier de typique : mobiliers spécifiques (tables, bibliothèques, classeurs, etc.) et fournitures (papiers, stylos, etc.) en sont les principaux éléments fonctionnels et décoratifs.

13 L'atmosphère particulière de ces lieux a été dépeinte par de nombreux écrivains. Balzac, Courteline, Perec sont de ceux-là. Ils apportent un regard sur les habitudes bureaucratiques qui modèlent les rythmes professionnels et plus encore qui transforment physiquement la figure de l'employé. Balzac $(1838,1841)$ évoque l'ennui, le besoin de s'occuper pour combler le temps. Il dresse un tableau pessimiste des conditions d'hygiène (lumière, poussière.) ; ce constat explique, pour lui, le besoin qu'éprouvent les employés de ne jamais s'attarder et de quitter leur bureau au plus vite. Ce lieu a toujours évoqué et continue à évoquer l'idée de monotonie, de tristesse, de travail ordinaire et routinier, de paperasserie et d'encombrement mais aussi de stress, de contrainte horaire, de poids de la hiérarchie. Soulignant leur aspect " anonyme ", Georges Perec décrivait ces espaces de travail comme des «lieux vagues encombrés de dossiers mal ficelés, de tampons, d'attachestrombones, de crayons suçotés, de gommes qui n'effacent plus, d'enveloppes jaunasses. où les employés généralement revêches vous renvoient "de bureau en bureau » en vous faisant remplir des formulaires, signer des registres et attendre votre tour »(1989: 90). Cette image 
stéréotypée, un peu passéiste, renvoie à celle du système bureaucratique et en constitue une mémoire. Ces représentations du bureau d'employé appartiennent à une idéologie de l'ordinaire. Qu'en est-il vraiment?

\section{Transformations d'un cadre de vie}

En dehors des ouvrages d'Elisabeth Pélegrin-Genel $(1994,1995)$, peu de textes abordent, à notre connaissance, d'une façon approfondie, la question de la transformation spatiale et décorative des bureaux en France. Au cours du $\mathrm{XlX}^{\mathrm{e}}$ et $\mathrm{XX}^{\mathrm{e}}$ siècles, nous passons progressivement d'une uniformisation à une personnalisation des bureaux. Si nos travaux ne nous permettent pas actuellement de dater ce passage, nous pouvons supposer que des changements significatifs s'opèrent à partir des années 1960 et 1970, suivant les mutations globales de la société française, et en particulier une tendance à l'individualisme. Cependant, l'impact d'une «tradition» organisationnelle (administration, entreprise paternaliste, etc.) rend, sans doute, les conditions de ce processus plus lent alors que l'émergence de nouveaux secteurs d'activités plus propices à l'innovation favorise, en revanche, des politiques et des formes de travail inédites et avec elle, des aménagements plus originaux et variés des locaux.

\section{De l'uniformisation des bureaux...}

Les premiers espaces de travail occupés par des employés sont plutôt collectifs et principalement masculins. À la fin $\mathrm{du} \mathrm{XlXe}$ siècle, les femmes qui travaillent sont secrétaires - personnels attachés au «chef de bureau » et symbole, par excellence de l'employée -, sténographes et dactylographes, demoiselles du téléphone, etc.. Le modèle d'agencement des bureaux ou plus exactement de l'espace de travail rappelle celui de l'usine. Ce modèle s'organise autour d'un principe de standardisation de l'environnement et de rationalisation des tâches que l'on pourrait rapprocher du modèle tayloriste, faisant du bureau, un espace impersonnel. Rendement, efficacité restent les points forts de cette organisation qui régente également le temps libre, le hors travail des employés; et si les bureaux sont en espace ouvert, c'est pour mieux en faciliter le contrôle. Bureau collectif d'employés ou pool de dactylographes, les lieux du travail se ressemblent par leur structure conformément aux règles d'agencement: plan de travail en enfilage et sur plusieurs rangs avec un poste de surveillance situé en surplomb sur une estrade ou à un angle visuel stratégique; le mobilier est identique - table, meuble ou casier de rangement, corbeille; le port de la blouse obligatoire accentue ce contexte d'uniformisation. La décoration reste sommaire. Les affaires personnelles sont pour une part rangées dans des vestiaires, ce qui présuppose le retrait, dans l'espace public, de tous les objets intimes. Ces vestiaires servent également selon les moments de la journée au rangement des tenues de travail. Pour le XlXe siècle. Honoré de Balzac évoque "des armoires et des endroits obscurs où chacun met l'habit de travail, les manches en toile, les gardevue, casquette, calottes grecques et autres ustensiles de métier; où se déposent les socques, les doubles souliers, les parapluies» (1841: 46). Encore en 1974, dans un hôpital parisien, comme nous l'avons noté sur une photographie, les bureaux de la gestion ont un caractère austère, accroché sur un mur, un calendrier et posés sur les bureaux, des piles de dossiers délimitant le plan de travail et marquant une frontière entre l'employé et le visiteur. L'uniformisation s'impose comme une norme difficile à contourner qui pourrait 
laisser supposer une dépersonnalisation des lieux. Pourtant, la présence d'objets quels qu'ils soient offre la possibilité d'un réagencement personnel même minime du plan de travail. Il suffit de déplacer instruments de travail, fournitures, cet acte étant une manière discrète, pour le salarié, de réordonner et de faire sien un territoire réglementé.

Bien que Balzac affirme qu'«à Paris, presque tous les bureaux se ressemblent» (1841: 41), insistant ainsi sur leur uniformisation, il propose au lecteur de saisir ces espaces et leur mode d'appropriation au regard des différentes personnalités qui les habitent et en croque des portraits pointus, décrivant les habitudes, les manies de chacun. Dans ces bureaux, les activités s'apparentent à celles de la maison : on y mange, on pouvait même y dormir jusqu'en 1913 (Gazier, 1941). L'uniformisation n'est donc pas synonyme d'absence de vie. Mieux on peut considérer que les employés prendront progressivement la liberté de réaménager leur espace de travail, comme bon leur semble. Par ailleurs, à partir des années 1950, designers et ergonomes se penchent sur ces lieux pour les rendre plus attrayants et plus confortables aussi. "Avec la création de lieux spécifiques de travail correspond la naissance d'un art de vivre des "employés » au bureau » (Pélegrin-Genel, 1995 : 32).

\section{...à une personnalisation}

17 Le décor des bureaux va prendre de nouvelles allures. Elisabeth Pélegrin-Genel note que "la décoration est perçue comme quelque chose de féminin, de superflu, qui n'a pas sa place dans la lutte économique» (1995: 110). Si l'on en croît ces propos, l'espace de travail doit se différencier de l'espace domestique dont la décoration serait un des éléments qualificatifs. Pourtant les ateliers d'hommes ne sont pas exempts d'objets décoratifs (affiches souvent à caractère érotique, photographies, calendrier...). La question porte plutôt sur la nature de la décoration et sur ce qu'une proximité féminine aurait changé dans le rapport au hors travail.

En effet, si les femmes ont toujours travaillé, elles accèdent, à partir de la fin de XlX siècle et plus sûrement dans les années 1960 à des emplois de bureau peu ou pas qualifiés. Cette féminisation de l'emploi, toutes fonctions confondues, conduit à formuler l'hypothèse d'une redéfinition des relations sociales et des conditions de travail dans les bureaux. Les femmes, peut-être plus sensibles à leur cadre de vie - elles ont longtemps été confinées au foyer, formalisant leur compétence dans l'entretien de leur intérieur domestique - n'auraient-elles pas, par un jeu de transfert, favorisé des modes d'appropriation spatiale personnalisés?

Avec la mixité des lieux professionnels, on peut dire aujourd'hui que les employés des deux sexes s'approprient les lieux de travail non seulement par un marquage matériel, décoratif de leur territoire (Fischer, 1989; Monjaret, 1996a) mais aussi par des activités extra-professionnelles : manger, jouer, faire la fête, etc. (Bozon, Lemel. 1990, Monjaret, 1997a, 2001b, 2001c). La privatisation de la sphère professionnelle est un fait difficile à contredire, bien que, dans certaines entreprises, le concept de «bureau zéro » ait pu être développé et mis en pratique, empêchant d'emblée l'appropriation stable d'un espace; dans ce cas, le caisson devient le territoire individuel minimal d'occupation et se définit par sa possible mobilité. Cette initiative aurait été mal reçue du personnel, se pliant contraint et forcé aux nouvelles règles de fonctionnement. Confortant nos thèses de l'imbrication des sphères, il est intéressant de signaler que l'année 2000 a vu le jour d'un nouveau concept venu des pays nordiques, le «bureau-cocon»: on doit désormais se 
sentir au bureau comme à la maison. Les locaux professionnels sont aménagés en conséquence: espace cuisine, espace ludique et de détente, fauteuil confortable sont proposés aux employés. Si l'on ne peut pas généraliser cette tendance, notons qu'en France, certaines agences de publicité en ont adopté les principes.

L'agencement du mobilier, le décor de bureau qu'impose l'institution sont souvent revus par l'employé qui cherche à s'entourer d'objets référentiels. Prenons le cas du siège d'une banque implantée à La Défense qui permet de souligner la dissension entre direction (encadrement) et salariés dans leur façon d'appréhender l'usage de l'espace. En 1996, lors d'une visite du site, un représentant de l'encadrement explique que les employés n'ont pas de "culture des bureaux » dans le sens où ils ne se tiennent pas à un aménagement rationnel. Il préconise leur formation à ce sujet ; des bureaux témoins sont installés et les employés ainsi guidés peuvent sélectionner leur mobilier constitué de pièces mobiles (modèle, agencement, couleur). Ils peuvent également se fournir en affiches encadrées dans une boutique située dans le hall principal de la société. Est-ce une façon de contrecarrer les initiatives personnelles et par là l'hétérogénéité du décor mural ? Estimation des besoins matériels et souci décoratif personnalisé orientent le choix des salariés qui vont, une fois installés, redéplacer leur ordinateur reconsidérant les principes ergonomiques, et très vite occuper l'espace avec des objets personnels et des plantes vertes. Il semble que, quelles que soient les conditions d'aménagement, l'individu veuille prendre possession des lieux en en modifiant le cadre initial. La «niche» individuelle, dans des espaces clos comme dans ceux plus ouverts, apparaît nécessaire dans la construction du bien-être des employés au travail.

21 Selon la politique de l'établissement ou selon les circonstances, ces pratiques soumises à un cadre législatif (règlement interne, code du travail) sont autorisées, tolérées ou interdites. Ainsi, prendre ses repas dans les locaux affectés au travail ou plus encore consommer de l'alcool peuvent être permis ou relever de la sanction. Quand l'espace est accessible au public, les règles sont plus strictes. L'écoute d'un poste de radio peut être interdite. Cette règle n'est toutefois pas toujours respectée. Un contexte de tensions dû à des perspectives de fermeture, comme nous l'avons observé dans des services administratifs hospitaliers, a conduit des agents à adoucir leur ambiance de travail par un fond sonore radiophonique ; la particularité de la situation a rendu, semble-t-il, aux yeux de la hiérarchie, ces initiatives tolérables.

22 S'il existe donc des directives institutionnelles, parfois l'obligation de se référer à son supérieur hiérarchique proche pour modifier son environnement de travail, l'individu n'hésite pas à réaménager son cadre de vie, contournant les règles pour s'ajuster à une nonne collective ou pour adapter une "niche écologique " à ses propres critères de confort. La marge d'autonomie des employés s'exprime, entre autres, dans cet espace de liberté. Pour le nouveau venu, la prise de possession des lieux se fera progressivement, suivant les codes du groupe de proximité ou s'en détachant afin de s'affirmer comme individualité. Cela pose la question de l'existence d'une culture commune des bureaux et du modèle, explicite ou implicite, à reproduire car, on le sait, le bureau d'un employé n'est pas celui d'un dirigeant. Plus somptueux, il est le symbole de son pouvoir, il est « le Saint des saints» (Perec, 1989: 90). Le bureau est à l'image de son occupant et réciproquement.

Dans tous les cas, le bureau reste un espace habité. La culture des bureaux doit donc se lire plus finement à travers toutes les logiques (économique, sociale, organisationnelle, affective...) qui coexistent autant qu'à travers les profils d'activités spécifiques qui 
s'observent dans l'entreprise. Le bureau renvoie à la personnalité, l'activité et la position hiérarchique de l'individu et apporte des indices sur la politique l'aménagement et de gestion humaine des établissements.

\section{Les objets ordinaires, objets d'une culture de bureau...} offerts par des collègues ou amenés volontairement au bureau par le salarié. En dehors du mobilier, ordinateurs, téléphones, papiers, bouilloires ou cafetières, plantes vertes, tableaux ou affiches, bibelots, etc. participent au décor. À ce titre, ils sont non seulement des indices mais aussi des analyseurs des normes et des pratiques de travail et de hors travail sur les lieux professionnels, des temps solitaires ou partagés, des formes de sociabilité. Nous avons choisi ici d'en présenter trois, représentatifs des usages au bureau.

\section{Pas de bureau sans papier}

Le papier, sous toutes ses formes, est l'une des caractéristiques des bureaux; la paperasserie en est le symbole. Rames de feuilles blanches, stocks de papier brouillon, post-it, chemises en carton, rapports ou livres, etc., leur masse est telle qu'elle est difficilement évaluable. Ces papiers qui encombrent les espaces de travail circulent, s'échangent, se trient aussi. Ils finissent immanquablement au rébus ou aux archives (Fischler, Lahlou, 1997). Sources ou supports d'activités comme l'écriture, le classement ou la lecture, ils font partie du décor. L'occupation de l'espace professionnel commence, pour certains, par l'accumulation progressive de documents papiers jusqu'à parfois former des piles, véritables contreforts, constructions remparts qui protègent le plan de travail du regard étranger, en délimitant matériellement l'espace. D'autres préfèrent faire table rase, classer au fur et à mesure de l'arrivée des documents, ranger soigneusement leur stock de fournitures. Chaque employé a sa manière de parfaire son environnement familier et de circonscrire son territoire.

Le papier n'est pas seulement support des productions (notes de service, rapports...) ou de pense-bête, il est aussi support iconographique. Les images restent un élément central dans la décoration du cadre de vie au travail. Papier peint imagé (plage et palmiers, coucher de soleil), tableaux, affiches, dessins (souvent d'enfants), collages, cartes postales, photographies (de famille, du groupe professionnel), photocopies de croquis humoristique, plus récemment images scannées installées sur l'écran de veille de l'ordinateur trouvent leur place dans les bureaux parce qu'ils disent les liens, cristallisent les souvenirs. Les acquisitions personnelles se mêlent aux biens de l'entreprise ou du groupe de travail. Punaisés, collés sur le mur, sur des panneaux, sur les portes, les documents recouvrent parfois entièrement ces surfaces. Accrochés des années, parfois non remplacés par un nouveau venu ou au contraire immédiatement enlevés, ils sont instruments de communication, de revendication, parfois d'autodérision. Ils permettent la production de discours. Avec le temps, le non-renouvellement des images fait oublier leur présence. On ne les remarque plus, elles font partie du paysage et d'une certaine manière, leur neutralité les sort d'un processus de réappropriation.

La façon dont chaque individu organise son panneau d'affichage est significative d'un rapport spécifique à l'espace de travail et à l'activité professionnelle. Dans le secrétariat d'un musée, une collection de cartes postales qui tapisse entièrement un panneau, 
souligne les relations sociales entretenues en dehors du temps de travail et montre le lien incessant entre la vie professionnelle et la vie privée. Dans un autre bureau, les panneaux qui offrent l'exposition d'images évoquant l'évasion et renvoyant aux hobbies de l'occupant. Dans celui d'un chercheur, des images souvenirs aux pense-bêtes, la composition du tableau montre qu'en s'éloignant du poste de travail, l'affichage perd de sa densité. Il s'agit d'avoir certains documents professionnels à porter de main. Ces images qui s'exposent, se donnent à voir dans les bureaux apportent des indices sur la personnalité des occupants, sur leur organisation de travail et sur leur construction d'un bien-être.

\section{Le téléphone, le lien permanent}

Le téléphone en est un autre exemple (Monjaret, 1996b, 1997b). Il est par excellence l'objet des contacts à distance, dans l'entreprise et avec l'extérieur. L'histoire du téléphone en fait d'abord un instrument de travail avant d'être un outil de la communication privée et domestique. Aujourd'hui, il brouille les frontières du domestique et du travail.

En interne, la relation avec un collègue est possible sans avoir à se déplacer ; l'employé qui déjoue ainsi les regards indiscrets, peut prendre des moments de détente téléphoniques échappant à une sanction que des déplacements abusifs appelleraient. Dans un bureau partagé, le téléphone peut provoquer des nuisances acoustiques ou des gênes dues aux interférences des sphères professionnelles et privées. Bien qu'il existe des textes internes qui réglementent l'usage des communications, interdisant souvent les appels personnels, le téléphone professionnel sert, durant la journée de travail, à communiquer avec les amis et avec la famille, conjoint ou enfants, parfois parents. Les femmes l'utilisent pour une gestion du domestique à distance (prise de rendez-vous chez le médecin, surveillance des devoirs des enfants, démarches administratives). Ces pratiques qui empiètent sur la sphère de travail conduisent le groupe de proximité à établir des normes de fonctionnement qui leur sont propres: ainsi, même si les communications privées sont interdites dans l'entreprise, l'organisation du familial au bureau est admise. En revanche, les communications relevant de situations intimes telles la maladie, un décès, une relation extra-conjugale qui installent un malaise entre collègues ne sont pas souhaitées. Il en est de même des appels personnels de longue durée. Ces conduites excessives seront vite sanctionnées. La mesure des différents degrés d'introduction de la sphère privée sert au cadrage des usages et à la régulation des normes au sein du groupe de travail de proximité.

\section{Décor végétal dans l'espace de travail}

Les plantes vertes, et le végétal en général, introduisent d'autres types de rapports sur les lieux de travail ; éléments de décoration, ils sont sources d'échange, de discussion entre collègues. C'est ainsi qu'ils occupent une place non négligeable dans les bureaux (Pélegrin-Genel, 1994). Pots ou bacs de plantes vertes (naturelles ou artificielles), affiches représentant des bouquets de fleurs, la campagne ou papiers peints paysages sont autant de formes végétales réelles ou imagées qui composent le décor. À l'hôpital où dans certains services les fleurs en pot ou coupées sont interdites, les posters ou dessins viennent pallier l'absence florale. Les bacs de plantes en créant des barrières naturelles 
peuvent servir à une recomposition spatiale. Les bouquets de fleurs sur le coin d'un comptoir sont les marques d'un accueil qui se veut chaleureux.

Parfois la verdure trouve un prolongement à l'extérieur grâce à la présence d'un jardin. Dans les hôpitaux, le jardin n'est pas seulement un espace public ; lieu de circulation, il est aussi un lieu de détente, de balade pour le personnel comme pour les malades, et par là même de rencontres. Le jardinier, ce professionnel de l'extra-professionnel, qui entretient les pelouses, sculpte les massifs, est plus qu'un artisan maîtrisant un savoirfaire. Il est un véritable artiste, jouant avec les formes, les couleurs, les espèces ; il aime à formaliser des messages, langage parallèle. Il manie l'écriture non sur le papier mais sur un sol terreux sur lequel il exerce l'art de la mosaïculture s'inspirant de nombreux motifs (logos, dates de commémoration, figurations animales, etc.). Le jardinier compose en outre des tableaux à l'aide de copeaux de bois colorés; par exemple à proximité de l'entrée d'une maternité, à même le sol, une cigogne est représentée transportant un nouveau-né dans un balluchon de linge blanc qu'elle retient par le bec. Cet ouvrage symbolise ce lieu des naissances.

32 Jardins, plantes vertes d'intérieur, fleurs coupées améliorent le cadre de vie professionnel; cet environnement naturel fait vivre les salariés aux rythmes saisonniers et calendaires : chrysanthèmes de la Toussaint, sapin de Noël, muguet de mai, gerbes de commémoration.

Les plantes vertes révèlent, elles, une culture de jardinage. L'arrosage, l'échange de boutures ou le prêt d'un pot à un collègue qui « a la main verte » durant les vacances, les cadeaux entretiennent ou créent des réseaux de sociabilité. On voit ici comment s'élaborent des relations d'entraide et d'échange au sein des locaux de travail en dehors du contexte directement professionnel. Ces éléments sur le végétal dans l'entreprise dévoilent la richesse d'un tel thème dans la compréhension métaphorique et empirique $\mathrm{du}$ lien social.

Tous ces objets utilitaires et/ou décoratifs qui renvoient à des pratiques particulières, professionnelles ou extra-professionnelles, sont révélateurs du fonctionnement de la vie en groupe ainsi que des relations que chaque salarié entretient avec son espace de travail, qu'elles soient normatives, neutres, personnalisées voire transgressives. Dans tous les cas, ils contribuent, par leur présence, à une forme de stabilité territoriale et morale qui, quand elle est remise en cause lors d'un déménagement, engendre d'une façon cyclique insécurité, tension, repli ou besoin festive des membres du personnel (Monjaret, 2001a). Cette situation de passage qui se construit sur des temps successifs (séparation, entredeux, appropriation) tend à souligner la place accordée par les individus à leur espace de travail et la crainte du nouveau : nouvelle organisation de travail, nouveaux locaux, car tout transfert implique un changement, parfois en complète rupture avec le vécu dans les anciens locaux. Les objets personnels (plantes vertes, photos, tableaux, bouilloires...) constituent un territoire minimal pour l'employé. Dans une situation de mouvement, ils deviennent des points de repère, une marque de stabilité, bien que leur intégration dans un nouvel espace en modifie l'ordre visuel initial. C'est en cela que les bureaux peuvent être considérés comme des espaces habités.

Plus qu'un espace de travail, le bureau est un « lieu » dans le sens où l'entend Marc Augé (1992). Pour lui, le lieu anthropologique se définit entre autres comme "celui au 'occupent les indigènes qui y vivent, $y$ travaillent, le défendent, en marquent les points forts, en surveillent les frontières» (1992 : 57). En effet, espace imposé par l'institution, le bureau est avant tout un 
espace de vie, c'est-à-dire un lieu occupé, parfois partagé, toujours réinvesti par les occupants qui en délimitent les frontières et lui donnent un nouveau caractère dans lequel le groupe (ou l'individu) se reconnait et affiche ses appartenances. La spécificité de chaque bureau se lit à travers les activités et des interactions qui s'y déroulent, une organisation spatiale particulière et une culture matérielle singulière. C'est ce qui nous fait dire que, s'il existe une culture des bureaux, elle ne peut être envisagée que dans une lecture plurielle. L'existence d'une culture dominée par les intentions directionnelles, souvent stéréotypale car motivée par des modèles commerciaux, ne doit pas nous permettre de faire l'économie des formes individuelles d'appropriations des espaces de travail pour définir la culture des bureaux, car cette dernière ne se réduit pas, loin de là, à celle imposée par une politique interne d'aménagement. C'est dans ce contexte complexe et dans ce contexte seulement que s'élaborent les identités au travail.

\section{BIBLIOGRAPHIE}

AUGE, M., Non-lieux, Introduction à une anthropologie de la surmodernité. Paris : Seuil. 1992.

BALZAC, H. de. Physiologie de l'employé. Paris : Ed. Aubert et Lavigne, 1841.

BALZAC, H. de, Les employés. Paris : Gallimard, 1985 (1838). BOZON, M., LEMEL Y., Les petits profits du travail salarié. Revue française de sociologie, 1990, n 30, p. 101-127.

COQUERY, N.. Patrimoine privé, patrimoine public : la conversion de l'hôtel aristocratique parisien en bureaux au XVIIIè siècle. In GRANGE, J., POULOT, D. (Ed.). L 'esprit des lieux. Le patrimoine et la cité. Grenoble : P.U.G., 1997, p. 377-390.

FISCHER, G.N., Psychologie des espaces de travail. Paris : Armand Colin, 1989.

FISCHLER, C, LAHLOU, S., Le bureau surchargé. Sciences Humaines, 1997, n 65, p. 42-45.

GAZIER, A., L'employé, ses droits, ses devoirs. Paris : P.U. F., 1941. GUIGO, D., Ethnologie des hommes des usines et des bureaux. Paris : L'Harmattan, 1994.

LAUTIER, F., Les transformations des espaces de travail et la mobilité urbaine. In BONNET, M., DESJEUX, D. (Ed.). Les territoires de la mobilité. Paris : P.U.F., 2000, p. 69-83.

MONJARET, A, « Etre bien dans son bureau ». Jalons pour une réflexion sur les différentes formes d'appropriation de l'espace de travail. Ethnologie française, 1996a, n 1, p. 129-139.

MONJARET, A., Les communications téléphoniques privées sur les lieux du travail : partage sexué des rôles dans la gestion des relations sociales et familiales. Traverse, 1996b, n 9, p. 53-63.

MONJARET, A., 1997a, La Sainte-Catherine, culture festive dans l'entreprise. Paris : Editions du C.T.H.S. MONJARET, A., Ethnographie des pratiques téléphoniques de « cadres » parisiens. Réseaux, 1997b, $\mathrm{n}^{\circ} 82-83$, p. 101-127.

MONJARET, A., Fermeture et transfert de trois hôpitaux parisiens. L'ethnologue, accompagnateur social. Ethnologie française, 2001a, $n^{\circ} 1$, p. 103-115. 
MONJARET, A., La fête, une pratique extra-professionnelle sur les lieux de travail. Cités, 2001b, n

- 8, p. 87-100.

MONJARET, A. (Ed.), L'alimentation au travail. In Consommations et Sociétés, 2001c, $\mathrm{n}^{\circ} 2$.

MOULINIE, V., La passion hiérarchique. Une ethnographie en usine. Terrain, 1993, n² 21,

p. $129-142$.

NIPPERT-ENG, C. E., Home and work. Chicago \& London : University of Chicago Press, 1996.

PELEGRIN-GENEL, E., L'angoisse de la plante verte sur le coin du bureau. Paris : EST éditeur, 1994.

PELEGRIN-GENEL, E., L'art de vivre au bureau. Paris : Flammarion, 1995. PEREC, G., L'Infra-ordinaire.

Paris : Seuil, 1989.

RHEIMS, M., Histoire du mobilier. In POIRIER, J. (Ed.), Histoire des mœurs. Paris : Gallimard

(Encyclopédie de La Pléiade), 1990, 1.1, p. 1075-1165.

\section{NOTES}

1. Si celle-ci a toujours existé, ce n'est seulement qu'aujourd'hui qu'apparaît une prise de conscience unanime et significative de ses effets sur les conditions de travail suscitant études et débats juridiques et organisationnelles.

2. Pour se référer à un terme utilisé dans le cadre des études sur les logements mais aussi parce que nous regardons ces locaux de la même manière que les espaces domestiques.

3. Bien que remanié, il utilise des extraits d'un texte, «L'officio : uno spazio vissuto e un nuevo campo per l'etnologo. L'esempio Francese ", à paraître dans Archivio Antropologico Mediterraneo, Italie.

4. En dehors des maisons de couture, banques, lycées, grands magasins etc., terrains d'une recherche sur la fête de sainte Catherine à Paris (Monjaret, 1997a), plusieurs enquêtes ont été effectuées depuis 1995 entre autres dans un bureau de la SNCF, deux musées, trois hôpitaux (Monjaret, 2001a) parisiens.

\section{RÉSUMÉS}

Les bureaux sont non seulement des espaces de travail mais aussi des espaces de vie dans le sens où les activités qui y sont effectuées ne sont pas systématiquement associées aux obligations professionnelles. Ces activités (travailler, manger, faire la fête, jardiner, décorer, etc.) ont un rôle dans l'inscription territoriale des salariés sur leur lieu de travail. À travers l'analyse des modes d'occupation et d'appropriation de ces espaces sur près d'un siècle, nous montrons comment s'est opéré le passage d'un aménagement uniforme à un aménagement qui mêle proposition de l'institution et décor personnalisé de l'employé. Dans ce contexte, l'usage des objets familiers (papiers, téléphone, plantes vertes, etc.) dévoile les fonctionnements organisationnels, hiérarchiques et communicationnels au travail et plus précisément bureaucratiques.

Offices are not only working spaces but also living spaces in the way activities that take place there are not systematically associated to professional obligations. These activities (working, 
eating, partying, gardening, decorating, etc.) have a role in the territorial appropriation of the workroom by the employees. Through the analysis of the modes of occupation and appropriation of these spaces over almost a century, we show how a uniform organization of offices turned into a more complex one. which mixes proposals from the institution and personalized decoration. In this context, the use of familiar objects (papers, telephone, house plant, etc.) reveals the «working of working «(organization, hierarchy, communication, and more precisely bureaucracy).

\section{INDEX}

Mots-clés : bureau, culture matérielle, appropriation, sphère privée, lien social

\section{AUTEUR}

\section{ANNE MONJARET}

Anne Monjaret, ethnologue, chargé de recherche au CERLIS (CNRS-Paris 5), développe une anthropologie des entreprises et des métiers. Considérant les bureaux et les ateliers comme des espaces habités, des lieux de vie, elle s'intéresse aux articulations entre le travail et le hors travail, entre les temps de stabilité et les temps de mobilité, en leur sein ; elle conduit actuellement des travaux sur les fermetures institutionnelles, accompagnées ou non d'une délocalisation, Mail : anne.monjare@paris5.sorbonne.fr 